\title{
Current Technique and Technology in Treatment of Extra-Articular Deformity: A Literature Review
}

Ravikant Kamal ${ }^{*}$

Department of Orthopedics, Independent Researcher, DLF Phase-4, Gurugram, 122001, India

*Corresponding author: Ravikant Kamal, Department of Orthopedics, Independent Researcher, DLF Phase-4, Gurugram, 122001, India, Tel: +91-9971882750; E-mail: ravikantkamal@gmail.com

Received Date: August 17, 2018; Accepted Date: August 24, 2018; Published Date: August 31, 2018

Copyright: (c) 2018 Kamal R. This is an open-access article distributed under the terms of the Creative Commons Attribution License, which permits unrestricted use, distribution, and reproduction in any medium, provided the original author and source are credited.

\begin{abstract}
Instead of advancement of technologies, extra-articular deformities are still considered a challenging case for total knee replacement surgery. Regular TKR procedures may not be sufficient to achieve the acceptable outcome in these cases. Pre-op deformity assessment in all three planes becomes very important in extra-articular deformed cases. Challenges related to surgical and imaging technique was reviewed in this article. Measurement technique and selection criteria were explored based on existing literature. On the technology front, surgeons have shown the trust in Patient-specific instrumentation and Navigation to overcome the challenges. Although there are various comparative studies exists in orthopedic literature but less has focused on extra-articular deformities due to its complex nature. We have explored the literature which included extra-articular deformity to compare both technologies in the context of user experience, post-operative alignment, logistics, and cost.
\end{abstract}

Keywords: Extra-articular deformity; Patient Specific Instrumentation; Navigation

\section{Introduction}

Presence of Extra-articular deformity (EAD) in knee is one the most complicated cases in TKA. Restoring mechanical axis which is considered as a core principal for TKA could be challenging to achieve in these conditions. Considering conventional surgery; Surgeon achieves anatomical axis by drilling intra-medullary canal using reference of femur center landmark which is 5 to $10 \mathrm{~mm}$ anterior to intercondylar notch and subsequently they adjust valgus angle which generally varies from $5-7^{\circ}$. In presence of EAD, it becomes difficult to drill due to bow in the mid-shaft region of femur. It has been suggested to drill laterally from the original femur center in case of bow [1]. Additional valgus error due to presence of extreme bow cases in conventional method may lead to restore false mechanical axis. To alienating these problems associated with conventional approach in EAD cases, surgeons have tried non-conventional approach. Computed assisted method which is frequently used in TKA in these cases can be categorized in two major technologies which are Navigation and PSI. Most of the current literature keeps both technologies in same brackets precisely in EAD cases. This article explores challenges associated with EAD considering all aspects of technique and technology involved in TKR.

\section{Extra-Articular Deformity}

Deformity present in femoral shaft proximal to medial epicondyle and distal to fibula head is defined as extra-articular deformity [1]. Extra-articular deformity can be uni-planer or multi-planer. It may occur in patients which have the history of trauma, childhood metabolic diseases like rickets or piget disease [2]. High tibial osteotomy can also result into EAD. Vitamin D and calcium deficiencies which is a primary cause of osteoporosis can also play a role in bowing [1]. Severe bowing can be seen as one of the reasons behind developing EAD. The relationship between $\mathrm{OA}$ and coronal bowing is very evident; according to one study, $20 \%$ of patient with OA may associate with severe coronal bowing [2]. It is evident that the Asian population are more prone to bowing deformity. Yau et al. have performed a study to mark bowing in the Chinese population, Out of 92 Knee, 58 (62\%) cases were found in the category of femoral bowing cases, which is considered very large [3]. Recent study on Indian population found that existence of inherent EAD and varus angulations exists in tibia which can affect the post-HKA angle if the angulations exceed more than $4^{\circ}[4]$.

\section{Radiological Evaluation}

Pre-op Planning has become the regular practice of TKA in over the years but $\mathrm{x}$-ray capturing technique in pre-op planning varies, mostly it can be classified as stitched $\mathrm{x}$-ray and full-length load bearing $\mathrm{x}$-ray. The process of stitched $\mathrm{x}$-ray limits the radiological exposure in hip, knee and ankle region. Eventually, these three regions will be stitched together using markers for ensuring the alignment. However this method can work in mild OA with no bowing cases but in severe bow condition, stitching method may hide essential bowing information. It is suggested by mulla ji et al. that in all routine TKA cases, full-length load bearing $\mathrm{x}$-rays adds more value than stitched $\mathrm{x}$-ray [5]. Evaluating valgus angle in patients is prior application of pre-op $x$-ray. The most frequent range of valgus angle varies between 5-7 but in case of lateral bowing and coxa vara cases it may increase and vice versa [5]. Chien Yen lee et al. has found that $32 \%$ Asian patient who is diagnosed with end-stage $\mathrm{OA}$ and genu varum deformity has a valgus angle outside of $(5 \pm 2)^{\circ}[6]$.

\section{Coronal deformity}

Proper scan orientation is very important in radiological evaluation. Neutral rotation of the limb should be cross-checked by aligning the patient's patella right in front of $\mathrm{x}$-ray and lesser trochanter should be visible while capturing $\mathrm{x}$-ray. Fundamental of coronal measurements 
revolves around measurement of HKA angle, femoral valgus angle, and mechanical axis deviations. HKA angle is defined as angulations between hip center, knee center and ankle center [7]. Non-diseased HKA angle may vary between $177 \pm 3^{\circ}$. Mullaji et al. have defined the protocol for measurement of coronal bowing [8]. If the coronal bowing angle is greater than $5^{\circ}$ then it can be considered as femoral bowing deformity. Moderate range of femoral bowing deformity falls between $10^{\circ}$ to $20^{\circ}$. Severe femoral bowing deformity should be considered when bowing exceeds from $20^{\circ}$ [9]. It is suggested to use full leg load bearing $\mathrm{x}$-ray for radiological diagnosis of severely deformed cases.

\section{Sagittal deformity}

Sagittal bowing can play a major role in implant mal-positioning. Malalignment in sagittal position may trigger notching issue. Implant partially placed in flexion can affect the extension gap and it may have an adverse effect on the polyethylene wearing. Implant partially placed in extension relative to femur may induce notching. Radiological evaluation for sagittal deformity in $\mathrm{x}$-ray could be challenging. There are several methods mentioned in literature for sagittal measurements. Chung et al. has suggested a new true lateral position in which patient placed diagonally and $\mathrm{x}$-ray beam tube was tilted to $15^{\circ}$. Using this method hip center could be easily recognizable and it's easier to identify the bow in bone. Also, he had defined to mechanical axis widely used in the navigation system by manufacturers. Mechanical axis-1 was defined as femoral head center and $1 \mathrm{~cm}$ anterior to the blumsaat line; where else, Mechanical axis-2 was defined as hip center and the point which is $65 \%$ posterior from the most prominent point and $35 \%$ from the anterior cortex region. He also defined anterior cortex axis as the line joining at $5 \mathrm{~cm}$ and $10 \mathrm{~cm}$ from knee line and distal medullary axis was defined as mid shaft line drawn at 5 and 10 $\mathrm{cm}$ proximal to knee line. Sagittal calculation of bow was measured as the angle between line joined at 5 and $10 \mathrm{~cm}$ proximal to knee line and line joined 10 and $15 \mathrm{~cm}$ distally from the hip center. The author also found a relationship between bow and leg length and bow and mechanical axis [10]. Apart from bowing, the presence of anvil osteophyte may also be one of the regions behind fixed flexion deformity [11].

\section{Axial deformity}

It has been observed that axial deformity in EAD cases is rarely documented in the literature. In order to achieve to appropriate rotation alignment in mild OA cases, it has become normal practice to give $3^{\circ}$ external rotation referencing posterior condyle line (PCL). The angle between the posterior condylar line and transepicondyle line has been considered as $3^{\circ}$ for most of the cases but exploring literature it is evident that the posterior condylar angle (PCA) varies in different population. The conventional approach of generalization of external rotation has been challenged by several recent researchers. Lalit maini et al. have found that for external rotation of knee in Indian population is $4 \mathrm{deg}$ [12]. Gurva reddy et al. also suggested on individualizing external rotation as a conclusion in CT based conclusion [13].

\section{D measurements}

Improvement of Preop Planning including CT and MRI in assessment practices triggers the 3D evaluation of deformity. There are free software resources available like ITK, Slicer etc which can create a $3 \mathrm{D}$ model using input data as CT/MRI. It may be useful input for assessing deformity but the accuracy of this model may be debatable. The process of extracting 3D model from CT/MRI data is called segmentation. It can be done manually, partially automated and fully automated. Two most frequently software used for manual segmentation are Mimics and Simpleware. These software can additionally use for picking the landmarks as well. There are various discussions available on the comparison of 3D landmark and 2D landmark [14]. Mostly PSI technology uses CT and MRI modality for their process. One of the advantages of $3 \mathrm{D}$ technology over $2 \mathrm{D}$ measurements is sizing prediction. The study has shown improvement in the accuracy with 3D based sizing technology [15].

\section{Surgical Approach and Decision Making}

Extra-articular deformity has been treated mostly with two approaches which are an Intra-articular correction and Extra-articular correction technique. Mulla ji et al. has categorized coronal deformity with less than $10 \mathrm{deg}$ as in general TKA without any influence of bow. Medial soft tissue release may be required until the achievement of equipoise in these cases. Removing the osteophyte meticulously always increases the chance of soft tissue balancing. Soft tissue release should be decided based on the assessment of whether the correction could be achieved fully or partially. In the case of varus, deep MCL and semi membranous tissue would be sacrificed. If the knee has hyperextension instability it is suggested to constrained with very conservative resection in both femur and tibia. Ligament balancing is a major challenge in case of deformity excess to $10^{\circ}$. These cases may persist in multi-planar deformity with lateral laxity and comparatively more osteophyte. Medial release could be more in these cases. The third category was cases where deformity exceeds more than $20^{\circ}$. Stepwise soft tissue release is advisable in these cases [5]. Intra-articular correction is most frequently used in EAD cases [5]. According to Piti Rattanaprichavej et al. Intra-articular correction technique could be used for deformity less than $20^{\circ}$ in femur and less than $30^{\circ}$ in tibia.

Extra-articular deformity in femur may be used when distal femoral cut may violate the collateral ligament and considering tibia when inter medullary axis passes out the tibia plateau [16].

Further extra-articular correction has been subdivided into singlestage procedure and double staged procedure. Several studies have shown the single staged process has a good outcome. B. Demir et al. have compared Oxford knee society score of 10 patients and observed satisfactory results. The average preoperative value was noted as 9 and post-op value was registered as 42 . Preop ROM was measured as $72^{\circ}$ which increased to $120^{\circ}$ post operatively [17]. Similar claim was shown in Zhang et al. studies where with 9 EAD patient, HSS (Hospital for special surgery) score was increased from 18.7 to 89.8 . ROM was also increased from $46.7^{\circ}$ to $100.6^{\circ}$ [18]. Hungerford et al. have set four criteria for decision making between intra-articular and extra-articular deformity correction: the magnitude of the deformity, the relationship of the deformity to the knee, the side of the deformity (varus or valgus), and whether the femur or the tibia is affected by the deformity [19].

\section{Technology and Its Application in EAD}

As the limitation of the conventional method, surgeons have shown interest in technologies like PSI and Navigation for the treatment of EAD. Both technologies work on the similar principal to restore mechanical axis preoperatively or intraoperatively using various image modality. 
Page 3 of 5

\section{Patient specific instrumentation}

PSI is a custom-made resection guide which is based on patient $\mathrm{CT} / \mathrm{MRI}$ images. It can be placed at the patient's knee and fix it through pin and subsequently, bone can be resected according to the surgeon's input. As it's a preoperative technique, Surgeon is required to be involved in preoperative planning phase. Surgeon has to decide the resection and angulations and then pre-op planner replicates that input in jig's design. The success of PSI depends on various factors. Steps involved in an image to instruments creation have various steps. Each step should be performed as per imaging protocol. The first step in PSI is to capture CT/MRI images. Both image modalities have its own advantage and drawbacks. Using CT modality one can capture smallest of bone detail accurately and conversion of CT into 3D model is easier using segmentation software. Landmark picking during preoperative planning is more accurate using CT extracted 3D model. However, there is always a concern of radiation is involved with CT imaging. To minimize the radiation it is advisable to expose only the certain part of extremity which is needed for creating the mechanical axis of femur like proximal and distal one-third of femur and tibia. Mostly slice thickness for PSI is limited to 1 to $1.25 \mathrm{~mm}$ in knee region. Slice thickness of Hip and Ankle region can be kept as $3-5 \mathrm{~mm}$. The accuracy of model depends upon the slice thickness and FOV (field of view) and various CT parameters; therefore, it is advisable to strictly follow the PSI protocol. Also, PSI technology has learning curve so surgeon training can help surgeon to achieve desired outcome. Restricting image capturing in certain region may have some drawback which is similar to capturing stitched $\mathrm{x}$-ray where information related to bowing cases might not appear.

\section{Navigation}

The first navigation system was used by Dr. William Barger in 1992 for THA, In 1997 Frederich Picard used for TKA but navigation was frequently used only in last one decade [20]. Navigation system can be categorized as an open and close system. Close system only supports the certain implant prosthesis while open system can support all prosthesis. There are three image modality exists in navigation: CT based, fluoroscopy based and Imageless implant. Patient co-ordinate system and Computer navigation system will be equi-orientated using bone registration done by surgeon by picking the required landmark by using pointer. Navigation uses intraoperative real-time technique to obtain mechanical axis. One of the striking differences from PSI in Navigation is capturing hip center. Hip center is determined dynamically by rotation the femur and ankle center was measured by 0.44 distance from medial malleolus. Once the entire landmark is picked according to the work instruction of navigation system, it can calculate the level of resection and angulations. Based on individual patient requirement surgeon can further modify the parameter [1]. Navigation system can be very useful in severely deformed cases as it is only system available which can provide dynamic assessment of knee and real-time size prediction. Study suggests the in EAD cases, navigation performs better in order to achieve a better outcome. [9] Since system eliminates the step of violating IM canal, chances of fat pulmonary embolism can be completely avoided. Post- op alignment has shown better result than conventional and PSI. Navigation has some disadvantage like cost, learning curve, increase surgical time and lack of long-term post-op follow up data [20]

\section{Comparison of PSI and Navigation in EAD}

Implant failure rate is up to $21 \%$ in EAD cases which is considerably higher than normal TKR cases. PSI and Navigation are expected to reduce the failure rate in EAD. It has been claimed that PSI reduces blood loss, which may reflect into faster recovery system. The surgical time was also reduced since steps involved in setup for femoral alignment guide was skipped [21]. Comparing with conventional approach PSI has better post-op alignment [22]. However, Literature on blood loss comparison is EAD was not found. Considering post-op alignment, Stefan et al. performed a study to compare the outlier between conventional, PSI and Navigation. Selection criteria of knee was set as which has preoperative deformity $>10^{\circ}$. PSI had minimum outliers, comparing all the three methods. [23]. Focusing on outcome both type of navigation: Imageless and Image-based is equally efficient in severe deformed cases. Keiichiro et al. dismissed the hypothesis of imageless do not perform better with severe deformed cases. In his study, he compared 17 varus case $\left(>20^{\circ}\right)$ and 81 varus cases $\left(<20^{\circ}\right)$ who underwent TKA using imageless navigation. All severe varus cases were able to correct in the acceptable range of $3^{\circ}$ [24]. Theinpont et al. conducted one meta-analysis which included 44 studies done during 2011 to 2015; 2866 knees underwent TKA using PSI and 2956 knees underwent using conventional process. As result, the relative risk of coronal malalignment of femur was lesser using PSI where else risk of tibia sagittal alignment was significantly higher than conventional approach. [25]. Chen et al. in his study discussed challenges with preoperative planning as surgeons have to devote additional time in reviewing planning and have to wait at least 5 to 7 days for PSI, which becomes very cumbersome for surgeons considering the volume of patient. Also, there is particular protocol which needs to be followed while using PSI jigs. If PSI jigs are CT based then surgeon has to avoid removing osteophyte and clear all the soft tissue in places where jigs need to be placed. On the other hand, compact navigation system like iASSIST need not to deal with preoperative planning and it can be used directly in the surgery without any lead time concerns [26]. It is difficult to perform study with EAD cases as compared to normal OA cases as EAD cases are very lesser in TKR practice. Emanuel et al. in his study have shown improved functional score of EAD cases operated by PSI. PSI may perform better compare to navigation as there are always challenge to pick a landmark in real-time especially in deformed cases [27]. Methodology to achieve mechanical axis in PSI and Navigation is fundamentally different, In PSI it is based on direct landmark picking where planner picks femur center and hip center on the other hand picking hip center in navigation is dynamic where surgeon rotates knee in a circular manner to get hip center. As navigation tracker need to be placed at the shaft region, chances of fracture and infection may be more in navigation compared to PSI, however, there are not any scientific literatures to support these claims.

\section{Cost comparison}

It is claimed in the study done by Hengjin et al. that Navigation is more cost-saving solution in long-term [28]. As per PSI is concerned, Emmanuel et al. has conducted one study to evaluate the costeffectiveness. According to the study, there is a huge indirect cost involved in PSI process. In the PSI process, Patient has to spend money on CT/MRI, travelling to image centers. Apart from this, there is cost involved in training to surgeon and patients, surgeon's involvement in preoperative planning review, transportation charge and sterilization of PSI jigs. Concluding all indirect cost involved in PSI is $40 \%$ of the total cost which is considerably huge [29]. Watters et al. compared the 
cost of conventional, PSI and Navigation; he found while comparing with conventional, PSI is not cost effective however comparing PSI with navigation system it can be considered as a cost-effective solution. PSI provides institute additional of 28 min compared to conventional and $67 \mathrm{~min}$ compare to navigation. These cost saving can impact the health care system more than implant related cost savings [30] However, there was no cost comparison study precisely focused on EAD cases. Considering complexity and expected longer surgical time PSI may not be cost effective in EAD cases but further studies may be required to reach into any conclusion.

\section{Discussion}

It has been observed that EAD cases are not very frequent in regular TKR practices; lack of comparative literature is very evident. There is need of more studies in this field to overcome the decision dilemma of technologies between PSI and Navigation. PSI could be useful in cases where the bone is osteoporotic and chances of fracture during surgery is more while comparing postoperative alignment Navigation performs better. Comparing surgical time, PSI has the edge in normal TKR but there is no evidence for EAD cases, more research would be needed to conclude the surgical timing discussion between Navigation and PSI. However PSI has been pitched as a time efficient solution of TKA, It needs to seen in a holistic way as PSI incorporates pre-operative surgical time of surgeon. This time can be further reducible after few surgeries when Preop Planner accustomed with particular surgeon approach and Preop Planning could be prepared based on the surgeon preference. Also, support of local manufacturing unit can reduce the logistic time to deliver it to the hospitals. PSI provides a Pre-operative 3D model which could be very useful complex cases. In recent advancement in $3 \mathrm{D}$ printing technology showing the impression that 3D printing technology may be more accessible and Hospitals are showing interest in 3D printing technology and we have started seeing the in-house 3D printing technology in hospitals. This could reduce the logistical cost and also it helps to operate patient with lesser lead time. Also, there is an initiative where researchers have used less costly imaging technology like $\mathrm{x}$-ray as in input creating PSI jigs which will also help to reduce the cost further [31].

\section{Conflict of Interest}

The authors report no conflicts of interest. The authors alone are responsible for the content and writing of this article.

\section{References}

1. Khanna V, Sambandam SN, Ashraf M, Mounasamy V (2017) Extraarticular deformities in arthritic knees-a grueling challenge for arthroplasty surgeons: An evidence-based update. Orthop Rev (Pavia) 9: 124-135.

2. Mullaji A, Shetty GM (2012) Correction of severe deformity in total knee arthroplasty: decision making and key technical considerations. Semin Arthroplasty 23: 27-30.

3. Yau WP, Chiu KY, Tang WM, Ng TP (2007) Coronal bowing of the femur and tibia in Chinese区: its incidence and effects on total knee arthroplasty planning. J Orthop Surg (Hong Kong) 15: 32-36.

4. Saibaba B, Dhillon MS, Chouhan DK, Kanojia RK, Prakash M, et al. (2015) Significant Incidence of Extra-Articular Tibia Vara Affects Radiological Outcome of Total Knee Arthroplasty. Knee Surg Relat Res 27: 173-180.

5. Mullaji AB, Shetty GM (2014) Deformity Correction in Total Knee Arthroplasty.
6. Huang TW, Peng KT, Lee M, Hsu RW, Shen WJ, et al. (2015) Variability of distal femoral valgus resection angle in patients with end-stage osteoarthritis and genu varum deformity: Radiographic study in an ethnic Asian population. Biomed J.

7. Cho M-R, Lee YS, Choi W-K (2018) Relationship between Lateral Femoral Bowing and Varus Knee Deformity Based on Two-Dimensional Assessment of Side-to-Side Differences. Knee Surg Relat Res 30: 58-63.

8. Mullaji AB, Marawar S V,Mittal V (2009) A Comparison of Coronal Plane Axial Femoral Relationships in Asian Patients With Varus Osteoarthritic Knees and Healthy Knees. J Arthroplasty 24: 861-867.

9. Lee C, Lin SJ, Kuo LT, Peng KT, Huang KC, et al. (2014) The benefits of computer-assisted total knee arthroplasty on coronal alignment with marked femoral bowing in Asian patients. J Orthop Surg Res 3;9: 122

10. Chung BJ, Kang YG, Chang CB, Kim SJ, Kim TK (2009) Differences between Sagittal femoral mechanical and distal reference axes should be considered in navigated TKA. Clin Orthop Relat Res 467: 2403-2413.

11. Lakdawala A, Ireland J (2005) The "Anvil" Osteophyte - A primary cause of fixed flexion of the knee? Knee 12: 191-193.

12. Maini L, Singh S, Kushwaha NS (2015) Radiographic analysis of the axial alignment of the lower extremity in Indian adult males. J Arthrosc Jt Surg 2: $128-131$.

13. Gurava Reddy A V., Mathur RK, Mugalur A, Eachempati KK, Reddy A (2016) Reference axes for optimal femoral rotational alignment in varus osteoarthritic Indian knees-A CT based study. J Clin Orthop Trauma 7: 215-219.

14. Hirschmann MT, Konala P, Amsler F, Iranpour F, Friederich NF, et al. (2011) The position and orientation of total knee replacement components: A comparison of conventional radiographs, transverse 2DCT slices and 3D-CT reconstruction. Bone Joint J 93-B: 629-633.

15. Ettinger M, Claassen L, Paes P, Calliess (2016). 2D versus 3D templating in total knee arthroplasty. Knee 23: 149-151.

16. Rattanaprichavej P, Laoruengthana A (2016) Total knee arthroplasty with extra- or intraarticular correction technique for arthritic knees with extra-articular deformity of the femur or tibia: A report of three cases. J Orthop Surg 24: 116-120.

17. Demir B, Özkul B, Saygılı MS, Çetinkaya E, Akbulut D (2018) Deformity correction with total knee arthroplasty for severe knee osteoarthritis accompanying extra-articular femoral deformity囚: the results are promising. Knee Surg Sport Traumatol Arthrosc.

18. Xiao-Gang Z, Shahzad K, Li C (2012) One-stage total knee arthroplasty for patients with osteoarthritis of the knee and extra-articular deformity. Int Orthop 36: 2457-2463.

19. Hungerford DS (2009) Extra-articular deformity is always correctable intra-articularly: to the contrary. Orthopedics 32.

20. Desai AS, Dramis A, Kendoff D, Board TN (2011) Critical review of the current practice for computer-assisted navigation in total knee replacement surgery: Cost-effectiveness and clinical outcome. Curr Rev Musculoskelet Med 4: 11-15.

21. Boonen B, Schotanus MGM, Kerens B, van der Weegen W, van Drumpt RAM, et al (2013) Intra-operative results and radiological outcome of conventional and patient-specific surgery in total knee arthroplasty: A multicentre, randomised controlled trial. Knee Surgery, Sport Traumatol Arthrosc 21: 2206-2212.

22. Daniilidis K, Tibesku CO (2013) Frontal plane alignment after total knee arthroplasty using patient-specific instruments. Int Orthop 37: 45-50.

23. Rahm S, Camenzind RS, Hingsammer A, Lenz C2, Bauer DE, et al. (2017) Postoperative alignment of TKA in patients with severe preoperative varus or valgus deformity: Is there a difference between surgical techniques? BMC Musculoskelet Disord 18: 1-8.

24. Maniwa K, Ishibashi Y, Tsuda E, Yamamoto Y, Inoue R, et al. (2013) Accuracy of image-free computer navigated total knee arthroplasty is not compromised in severely deformed varus knees. J Arthroplasty 28: 802-806. 
Citation: Kamal R (2018) Current Technique and Technology in Treatment of Extra-Articular Deformity: A Literature Review. J Bioengineer \& Biomedical Sci 8: 253. doi:10.4172/2155-9538.1000253

Page 5 of 5

25. Thienpont E, Schwab P-E, Fennema P (2017) Efficacy of Patient-Specific Instruments in Total Knee Arthroplasty: A Systematic Review and MetaAnalysis. J Bone Joint Surg Am 99: 521-530.

26. Yao C, Xu X, Jiang Q, Shi D (2017) Comparison of patient-specific instruments with the computer-assisted navigation in total knee arthroplasty for osteoarthritis in a patient with femoral fracture malunion. Ann Jt 2: 2-2.

27. Thienpont E, Paternostre F, Pietsch M, Hafez M, Howell S (2013) The Knee Total knee arthroplasty with patient-specific instruments improves function and restores limb alignment in patients with extra-articular deformity. Knee 20: 407-411.

28. Dong H, Buxton M (2006) Early assessment of the likely costeffectiveness of a new technology: A Markov model with probabilistic sensitivity analysis of computer-assisted total knee replacement. 2: 191-202.

29. Thienpont E, Paternostre F, Van Wymeersch C (2015) The indirect cost of Patient-Specific Instruments. Acta Orthop Belg 81: 462-470.

30. Watters TS, Mather RC 3rd, Browne JA, Berend KR, Lombardi AVJ, et al. (2011) Analysis of procedure-related costs and proposed benefits of using patient-specific approach in total knee arthroplasty. J Surg Orthop Adv 20: 112-116.

31. Karade V, Ravi B (2015) 3D femur model reconstruction from biplane Xray images: a novel method based on Laplacian surface deformation. Int J Comput Assist Radiol Surg 10: 473-485. 